\title{
Mechanisms of microbe-immune system dialogue within the skin
}

\author{
Nonhlanhla Lunjani $^{1,2} \cdot$ Sinead Ahearn-Ford ${ }^{2} \cdot$ Felix S. Dube ${ }^{3,4} \cdot$ Carol Hlela $^{1} \cdot$ Liam O'Mahony $^{20,5,6}$
}

Received: 12 January 2021 / Revised: 9 April 2021 / Accepted: 26 April 2021 / Published online: 15 May 2021

(c) The Author(s) 2021. This article is published with open access

\begin{abstract}
The prevalence and severity of dermatological conditions such as atopic dermatitis have increased dramatically during recent decades. Many of the factors associated with an altered risk of developing inflammatory skin disorders have also been shown to alter the composition and diversity of non-pathogenic microbial communities that inhabit the human host. While the most densely microbial populated organ is the gut, culture and non-culture-based technologies have revealed a dynamic community of bacteria, fungi, viruses and mites that exist on healthy human skin, which change during disease. In this review, we highlight some of the recent findings on the mechanisms through which microbes interact with each other on the skin and the signalling systems that mediate communication between the immune system and skin-associated microbes. In addition, we summarize the ongoing clinical studies that are targeting the microbiome in patients with skin disorders. While significant efforts are still required to decipher the mechanisms underpinning host-microbe communication relevant to skin health, it is likely that disease-related microbial communities, or Dermatypes, will help identify personalized treatments and appropriate microbial reconstitution strategies.
\end{abstract}

\section{Introduction}

Recent decades have seen a rapid increase in chronic inflammatory disorders due to inappropriate or misdirected immune responses accompanied by insufficient development of immune regulatory networks. It is generally accepted that changes in environment, lifestyle and dietary factors may play a role in the miseducation or deficient training of the immune system [1, 2]. In particular, factors that negatively impact microbial diversity and metabolism are thought to dramatically influence mechanisms of immunological tolerance [3]. An enormous variety of microbes colonize body surfaces and these microbes are

Liam O’Mahony

liam.omahony@ucc.ie

1 Department of Dermatology, University of Cape Town, Cape Town, South Africa

2 APC Microbiome Ireland, University College Cork, Cork, Ireland

3 Department of Molecular and Cell Biology, Faculty of Science, University of Cape Town, Cape Town, South Africa

4 Institute of Infectious Disease \& Molecular Medicine, University of Cape Town, Cape Town, South Africa

5 Department of Medicine, University College Cork, Cork, Ireland

6 School of Microbiology, University College Cork, Cork, Ireland organized within complex community structures, utilizing nutrients from other microbes, host secretions and the diet. Modern lifestyles, medications and social interactions have fundamentally altered and disrupted the human microbiome metacommunity and, as a consequence, risk of immunemediated diseases [4]. The mechanisms that contribute to the intimate and sophisticated inter-kingdom dialogue that maintains a stable environment with important beneficial physiological, metabolic, and immunological effects on the host are being intensely investigated by many research groups across the world. Although exposed to modification by the external environment, human skin actively regulates microbial colonization and microbial entry into dermis/ subcutis. Microbes interact with each other and with host cells, including keratinocytes and immune cells, in turn influencing skin homoeostasis (Fig. 1). Some of the host immune functions that are influenced by the skin microbiome include promotion of host defence networks against pathogens, control of inflammation, and education of adaptive immune pathways [5, 6]. Commensal skin microbiota can directly inhibit colonization and invasion by pathogenic microbes or opportunistic microbes. For example, Staphylococcus epidermidis (S. epidermidis is one of the most abundant commensal species in the skin) can stimulate keratinocyte antimicrobial peptides production, as well as inhibit inflammatory cytokine release and inflammation during wound healing [7]. In this review, we will 


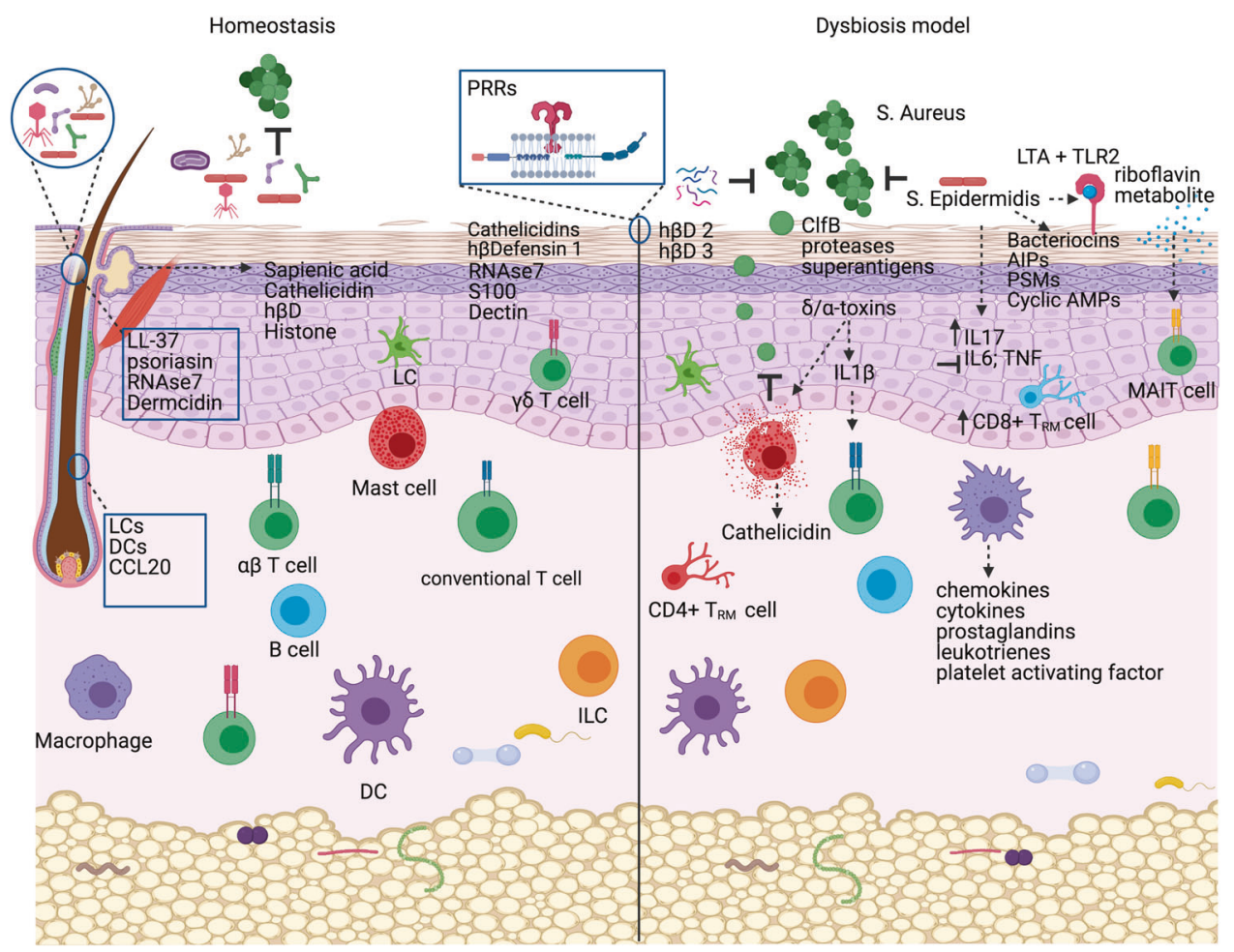

Fig. 1 Microbe-microbe and microbe-host interactions on the skin. Diverse microbes on the skin surface and hair follicles interact with each other such that they limit the proliferation of pathogenic organisms. Microbes influence the growth of other microbes via secretion of bacteriocins, auto-induced peptides (AIPs), phenol soluble modulins (PSMs) and cyclic anti-microbial peptides (AMPs). Keratinocytes inhibit microbial growth by constitutively secreting antimicrobial peptides such as cathelicidin and human beta defensins (h $\beta$ Ds). Pattern recognition receptors (PRRs) recognize microbial structures to induce appropriate innate immune responses. Lipotechoic acid (LTA) from Staphylococcus (S.) epidermidis is recognized via

summarize some of the key recent findings that identify novel mechanisms relevant to the unique features of host-microbe interactions in the skin and discuss the potential for novel preventative and therapeutic approaches using microbes or microbial metabolites for skin health.

\section{The importance of the skin habitat}

The composition and function of human skin is such that it does not only form a barrier to the external environment but serves as dynamic ecosystem consisting of living and nonliving components that dictate the local environmental and nutrient conditions of the skin surface. Components of this ecosystem are highly interactive and function together as a sophisticated system. Different methodologies (culture and non-culture based) have provided precision and resolution in surveying skin microbial communities, confirming the presence of the bacterial, fungal, viral and mite toll-like receptor 2 (TLR-2). Mucosa-associated invariant T (MAIT) cells specifically respond to microbial-derived riboflavin metabolites. Innate cells such as Langerhans cells (LCs) and dendritic cells (DCs) sample microbial antigens within the hair follicle, while secretion of chemokines including chemokine (C-C motif) ligand 20 (CCL20) control the recruitment of lymphocyte subsets. Dysbiosis is associated with overgrowth of microbes such as $S$. aureus, which employs clumping factor $\mathrm{B}$ (ClfB), toxins, proteases and superantigens to colonize the skin and induce damaging inflammatory responses. Figure created with BioRender.com.

communities in the skin. The emerging picture indicates that the skin harbours a diverse population of microbes whose composition is largely determined by site-specific physiological factors, such as moisture and sebum content $[8,9]$.

At the forefront is the highly keratinized epidermis, the result of a specialized differentiation process of keratinocytes (the main cell type in the epidermal barrier). The uppermost layer of the epidermis, the stratum corneum, harbours a rich diversity of microbes, contributing to the barrier properties of the skin [10]. Current detection techniques have shown that microbes reside not only on the external interfollicular epithelial surface but also on the entire skin appendage surface and even below the basement membrane, extending to the dermis and dermal adipose tissue [11]. Appendage structures including sebaceous glands, hair follicles, eccrine ducts, and apocrine ducts maximize epithelial surfaces for microbial attachment and colonization, suggesting that the epithelial surfaces of skin 
appendages are relevant interfaces for cross-talk between microbes and the host [12].

Sebum, a lipid-rich substance secreted by the sebaceous glands lubricates the hair and skin. The hydrolysis of sebum by commensal microbes generates free fatty acids such as sapienic acid, which work to control microbial colonization along with sebocyte-derived anti-microbial peptides (AMPs) such as cathelicidin, $\beta$-defensins and antimicrobial histones [13]. Eccrine sweat (water, salt and electrolytes) secreted directly onto the skin surface, contributes to the acid mantle of the skin, creating an environment that limits the composition of microbes that can survive and proliferate. The density of eccrine sweat glands impacts the microbial colonization of the skin [14]. Cutibacterium acnes is lipophilic and found in abundance in sebaceous skin sites. C. acnes is also responsible for acne vulgaris, producing various adhesions, toxins and inflammatory mediators. Staphylococcal species are found in moist skin niches, and are halotolerant organisms that have evolved to use urea found in sweat as a nitrogen source. Certain Staphylococcus species, such as pathogenic $S$. aureus strains, employ multiple mechanisms in order to colonize the skin. They are able to (i) produce adhesins such as clumping factor B that promote bacterial adherence to corneocytes, (ii) disrupt the epidermal barrier due to $\alpha$-toxin and various extracellular proteases and (iii) activate inflammatory responses by staphylococcal superantigens $[15,16]$.

Human skin has approximately 2 million hair follicles. This dermal appendage is home to a unique and complex microbiota including bacteria, fungi, viruses (including bacteriophage) and mites. The follicles form tissue columns within human skin that directly link the skin environment and its surface microbiome with all cutaneous layers, thereby making the skin the largest epithelial surface in the human body for efficient microbe-host immune interactions and an ideal habitat that favours microbial survival. This is kept in check by an inhibitory environment in the hair follicle whereby bacterial metabolites induce AMPs such as cathelicidin LL-37, psoriasin, RNAse 7 and dermcidin. Within the hair follicle, both Langerhans cells and CD11b+ type 2 conventional dendritic cells (cDC2s) acquire and present topical antigen to $\mathrm{T}$ cells, and the IRF-4-dependent $\mathrm{cDC} 2 \mathrm{~s}$ are required for $\mathrm{T}$ cell priming and $\mathrm{LAP}+$ regulatory $\mathrm{T}$ cell (Treg) expansion [17]. Young mice have reduced Treg cells in the absence of hair follicles. The niche provided by hair follicles accommodates coagulase-negative Staphylococci (CoNS) species such that these microbes appear to stimulate hair follicle production of chemokine (C-C motif) ligand 20 (CCL20), which is chemotactic for Treg migration into skin [18]. These resident microbes appear to be necessary for differentiation of skin stem cells and establishment of immune tolerance to commensal microbes. The outer root sheath keratinocytes are able to mobilize inflammatory cells in the event of microbial dysbiosis [19].

The dermis was previously thought to be devoid of a microbial community in the absence of barrier defects. However, this concept has been challenged. DNA from Proteobacteria, including Burkholderiales and Pseudomonadales, as well as Actinobacteria, have been detected in the subepidermal compartments [20]. Notably, dermal bacterial sequences were dissimilar to those detected on the skin surface and there was no evidence to support the translocation of bacteria via phagocytic cells into the subdermal compartment. The viability of the dermal-associated microbes is yet to be confirmed, but microorganisms need not be alive to exert effects on the host immune system, as discussed in more detail below.

\section{Microbe-microbe interactions on the skin}

Bacterial species colonizing the same ecological niche interact extensively with each other as they compete for nutritional resources. Bacteria may sequester or consume nutrients in a niche preventing their competitors from accessing them, produce antimicrobials that synergise with host-derived AMPs to inhibit the growth of their competitors, or produce factors that interfere with the virulence signalling pathways of their competitors [21-23]. These competitive interactions are important in shaping the composition and diversity of microbial communities and ecosystems, which have important consequences to skin health.

S. aureus overabundance, accompanied by a concomitant decline in microbial richness and diversity (especially protective staphylococci) is associated with atopic dermatitis (AD) pathogenesis [24, 25]. Intraspecies competition among Staphylococci can inhibit pathogenic strains such as $S$. aureus, by production of (i) bacteriocins, and (ii) autoinducing peptides, which inhibit accessory gene regulator (agr) quorum sensing systems that controls production of virulence factors [26]. The peptides phenol soluble modulin (PSM) $\gamma$ and PSM $\delta$, produced by $S$ epidermidis, limit survival of $S$. aureus on the skin surface. These PSMs cause membrane leakage and membrane perturbation in bacteria, suggesting that these peptides function by a mechanism similar to that of human AMPs. $S$ epidermidis strains are also capable of producing specific serine proteases that interfere with $S$. aureus biofilm formation. Staphylococcus hominis strains can produce $S h$-lantibiotics $\alpha / \beta$ - a class of cyclic AMP that contain lanthionine and methyllanthionine. Staphylococcus lugdunensis also produces the cyclic peptide lugdunin that inhibits S. aureus [26-29]. An unidentified antimicrobial factor from Corynebacterium pseudodiphteriticum can inhibit $S$. aureus growth and colonization as well as biofilm formation on anterior nares [22, 30]. 
In addition to antimicrobial factors, signalling inhibitory molecules produced by skin commensal microbes and nasal microbiota can inhibit functioning of the $S$. aureus agr quorum-sensing system in a process called quorum quenching [31-33]. Quorum quenching is associated with reduced $S$. aureus virulence, including inhibition of biofilm formation, haemolytic toxin production and enhanced host immune responses against $S$. aureus in murine skin infection models. In an inflammatory skin disease murine model, treatment with Solonamide B reduced $S$. aureus RNAIII and delta-PSM expression, which was associated with reduced mast cell degranulation and proinflammatory cytokine production [34].

Using $S$. aureus as a model pathogenic microbe, these findings highlight the importance of commensal bacterial species in the prevention of $S$. aureus colonization and pathogenic outcomes during $S$. aureus infections or flareups. Skin and nasal commensal microbes produce antimicrobials that limit $S$. aureus growth and colonization, but the relative abundance of these commensals and the potency of their antimicrobial activity against $S$. aureus are often reduced in AD. However, it is too simplistic to suggest that $S$. aureus is the only skin microbe that might negatively influence the skin in patients with AD. Even selected bacterial strains from presumptive commensal species can have detrimental effects. Specific $S$. epidermidis strains can be deleterious to the skin barrier through protease activity that is similar to S. aureus [35]. Proteolytic activity was mediated by secretion of an extracellular cysteine protease A (EcpA) controlled by the agr quorum sensing system.

In summary, complex niche-specific ecological networks govern the relative abundance and activity of skinassociated microbes, which have important consequences for skin homoeostasis and health. In addition to $S$. aureus, other potentially damaging microbes such as $C$. acnes are now being shown to be controlled by non-pathogenic microbes [36]. Intervention strategies designed to modify entire communities within a given niche, rather than simply targeting a specific individual microbe, will likely be more effective in the long term.

\section{Microbe-immune system interactions on the skin}

Skin microbiota composition changes during development and is age dependent. In addition, microbial composition is related to changes in host physiology and the influence of external environmental factors. It is also evident that the skin microbiota adapts to prevailing physiological and immunological environment of the sites they inhabit and in turn has unique functional influences on immune maturation and activity in the ecological niches they occupy [37].
While significant skin site specificity is evident, the microbiome usually remains stable at each site over many years.

Neonatal skin is structurally similar to adult skin reaching adult-like maturity at 34 weeks gestation but with very different physiological and immune activity. The skin surface is extensively colonized immediately postpartum with maternally and environment-acquired bacterial strains. This composition is displaced by successful acquisition of further environmental microbes during skin maturation. Early life skin microbial acquisition events and encounters may have long-term health implications through modulation of host immunity and microbe-microbe interactions. In particular, the post-natal period is very important for the development of immune tolerance. Levels of FoxP3 + Treg cells coincide with S. epidermidis colonization. Continued exposure to commensal microbes modulates host immune and epithelial cell production of AMPs, cytokines and can inhibit proinflammatory immune activation [38, 39]. Adaptive immune responses in human skin develop during early childhood, however, the neonatal skin is skewed towards antiinflammatory responses as it's abundantly populated by Treg cells. Similarly, mice colonized with benign microbial strains in the neonatal period preferentially induce tolerogenic immune responses in skin and gut, while reduced Treg cell numbers have been observed in the skin of young mice raised under germ-free conditions [40, 41].

The early life human skin microbiome stabilizes at about 3 years of age, but then goes through marked changes at the onset of puberty due to hormonal influences on skin physiology, notably sebum production that supports a lipophilic microbiota. Skin physiology in the elderly is altered by several host factors such as hormones and diminished cellular metabolism including immunosenescence. This leads to shifts in microbial composition of the skin. A decline in Propionibacteria correlates with a decline in sebum production. In contrast, Archaea relative abundance increases with lower sebum levels. Commensal fungi such as Malassezzia seem to remain stable with advancing age.

The beneficial effects on host immune maturation mediated by diverse environmentally acquired microbial exposures have been suggested by several studies. It has been shown that human skin shares numerous common bacterial taxonomies with soil microbes [42]. Recently, environmental biodiversity was deliberately manipulated to examine its effects on commensal skin microbiome and the immune system in young children. The intervention entailed enrichment of urban daycare centre yards for 28 days with segments of forest floor, sod, planters for growing annuals, and peat blocks for climbing and digging. Increased microbial biodiversity was associated with changes in the skin and gut microbiota of children, which, in turn, were related to changes in plasma cytokine levels and regulatory 
T-cell frequencies [43]. Specifically, the intervention was associated with a shift toward a higher ratio between plasma Interleukin-10 (IL-10) and IL-17A cytokine levels and a positive association between Gammaproteobacterial skin diversity and regulatory $\mathrm{T}$ cell frequencies in blood, suggesting that the intervention may have stimulated immunoregulatory pathways. After the trial, children in the intervention daycare centre had more diverse skin Proteobacterial and Gammaproteobacterial communities than children in standard daycare settings. These results demonstrate how environmental biodiversity can promote or prevent the loss of skin bacterial species.

In addition to the epidermis providing a formidable physical barrier and a mutually beneficial habitat for selected microbes, it also supports well-choreographed immune functions. AMP secretion and expression of Pattern Recognition Receptors (PRRs) by keratinocytes regulate microbial density and community composition. Microbes interact with keratinocytes to limit the potential overgrowth of pathobionts. Keratinocytes express several PRRs that are able to distinguish a wide variety of microbial components, including Toll-like receptors (TLRs) bacterial peptidoglycan sensing Nod-like receptors (NLRs), and NLR pyrin domain-containing proteins that sense viral, fungal and self-proteins [44]. Keratinocyte expression of RIG-Ilike receptors (RLRs) enable detection of viral RNA, whereas antifungal immunity is tailored by non-TLR signalling such as dectin-1 [45]. RNAse 7 is constitutively released by keratinocytes and has potent antimicrobial activity on a broad spectrum of microorganisms. Damageassociated molecular patterns such as the $\mathrm{S} 100$ proteins on keratinocytes can inhibit microbes, with S100A7 also exhibiting a chemotactic function [46]. In addition to the direct microbe-microbe interactions described above, commensal organisms can also augment host defenses. For example, PSM $\gamma$ and $\delta$ from $S$. epidermidis, which have direct antimicrobial effects on $S$. aureus, also activate TLR2 and enhances tight junction barrier function, induces keratinocyte-derived AMP, induces IL-17 production while inhibiting inflammatory cytokines such as IL-6 and TNFalpha [47]. Another S. epidermidis-derived TLR-2 ligand, lipotechoic acid, inhibits proinflammatory signals following epithelial injury and enhances $\mathrm{CD} 8^{+}$skin-resident $\mathrm{T}$ cell functions. These $S$. epidermidis specific $\mathrm{CD}^{+} \mathrm{T}$ cells express immunoregulatory and tissue repair gene signatures [5]. Tissue resident memory $\mathrm{T}$ cells $\left(\mathrm{T}_{\mathrm{RM}}\right)$ have been identified in human skin in areas of the previous infection. $\mathrm{CD} 8+\mathrm{T}_{\mathrm{RM}}$ cells locate themselves in the epidermis and $\mathrm{CD} 4+\mathrm{T}_{\mathrm{RM}}$ cells in the dermis, which corresponds with similar $\mathrm{T}_{\mathrm{RM}}$ migration patterns observed in mouse studies of HSV-1 infection [48]. The extent to which specific commensal microbes influence $T_{R M}$ cells has yet to be determined.
Microbes have direct and indirect effects on the skin barrier. Filaggrin, a key skin barrier protein with an important role in $\mathrm{AD}$ pathogenesis, and epidermal lipids influence microbial growth. Overgrowth of certain pathogens stimulate cytokine and chemokine secretion by keratinocytes that then direct respective innate effector cell and adaptive immune cellular function. Damaging the skin barrier can lead to epicutaneous senitization via the TSLPbasophil-IL-4 axis [49]. The skin microbiome negatively or positively influences the skin epithelial barrier [50]. A characteristic microbiome signature has often been described for $\mathrm{AD}$ and this pattern was recently shown to associate with the expression of type 2 inflammation pathway genes such as IL-4R, C-C motif chemokine receptor type 4 (CCR4), and C- C motif chemokine 22 (CCL22) in lesional compared with non-lesional skin [51]. Biological processes related to keratinization were the key host response pathways identified in the dry/lipid-poor microenvironments such as the upper leg. S. aureus abundance was associated with enrichment for genes important for extracellular matrix organization and leucocyte migration to the skin. In contrast, S. epidermidis was associated with enrichment for genes related to epidermis development.

Specialized antigen-presenting cells such as epidermal dendritic cells known as Langerhans cells are located above the basal keratinocyte layer. Their dendrites are able to project towards the horny layer in order to sample microbial components. It has been shown that they efficiently prime immune responses to $C$. albicans and $S$. aureus thereby inducing requisite effector T-cell responses. Langerhans cells are able to sample bacterial toxins, and favour specific humoral immune responses whilst ensuring that the epithelial barrier remains intact. CD1c + dendritic cells (DCs), CD14+ dendritic cells (DCs of monocyte origin), and CD141+ DCs have a role in antiviral immunity and present antigen for CD8 + T-cell responses. Following pathogen detection, activated skin macrophages rapidly produce chemoattractants, cytokines, prostaglandins, leukotrienes and platelet activating factor [52].

Mast cells (MCs) are located in the upper dermis and contribute to maintaining microbiome-tissue homoeostasis [53]. They are able to produce AMPs such as cathelicidin and have direct bactericidal activity. MCs can recognize microbes through different mechanisms including direct binding of pathogens or their components to TLRs, NLRs, RLRs, and activation of complement receptors. Once microbes activate these receptors, inflammatory mediators are released. These mediators contribute to effective antimicrobial immune responses. In $\mathrm{AD}, S$. aureus peptidoglycans recruit MCs and $S$. aureus delta toxins induce MC degranulation, which damage the epithelial barrier and further promote innate and adaptive inflammatory responses [54]. S. aureus $\alpha$-toxins can also induce IL- $1 \beta$ production 
from skin monocytes, which may further promote effector $\mathrm{T}$ cell responses.

Commensal microbes control mucosal-associated invariant T (MAIT) cells. These are an evolutionarily conserved T-cell subset, which represents the most abundant T-cell subset recognizing bacterial compounds [55, 56]. They react to many bacterial species through $\mathrm{T}$-cell receptor (TCR)-mediated recognition of metabolites derived from the vitamin B2 biosynthetic pathway. MAIT cells reside in peripheral tissues during homeostatic conditions with the microbiota seemingly a strong determinant of MAIT cell numbers. The MAIT17 subset, compared to MAIT1 cells, home preferentially to barrier tissues such as the skin, lung and gut. MAIT cells are important for the clearance of bacterial infections [57]. Their role in defence against viral infection has also been noted. In addition to anti-microbial activity, MAIT cells improve wound healing in the skin and are also thought to regulate lung and intestinal epithelial integrity, suggesting a role in epithelium homoeostasis through bi-directional interactions with the local microbiota. In keeping with these observations, blood MAIT cell frequency is modified in inflammatory disease where microbial dysbiosis is a pathogenic feature [58]. As MAIT cell development in mice is restricted to early life, it is possible that dysregulated immune-microbe cross-talk during childhood may negatively affect MAIT cell function throughout life.

\section{Human clinical trials investigating microbial treatments for skin conditions}

Over the past decade, a number of clinical trials evaluating the efficacy of probiotic and prebiotic interventions in dermatologic diseases have been published with mixed results (summarized in Table 1).

The potential for probiotics to treat paediatric AD has been a focal point of research. A number of randomized controlled trials (RCTs) have reported a reduction in disease severity and inflammatory markers following administration of oral probiotics to children with AD [5967]. Certain large studies have observed significant preventative effects of oral probiotic supplementations for paediatric $\mathrm{AD}$, when given both pre- and postnatally to mothers and their infants [68-70]. Conversely, other trials have noted no differences between oral probiotics and placebo in preventing or improving the clinical presentation or inflammatory markers seen in children with AD [71-75]. Several studies have assessed the combination of probiotics and prebiotics (synbiotics) in this AD population and reported decreases in disease severity [76-78]. Interestingly, studies using prebiotics alone had some activity against $\mathrm{AD}$ [76, 79]. However, other trials have reported the significant lack of synbiotic activity in this population [80, 81].

The use of oral probiotic interventions in adult acne has similarly gained significant attention. Trials have demonstrated clinical improvement using probiotics alone and in combination with standard treatment [82-84]. Nevertheless, one trial reported that probiotics alone provided little benefit, yet reported significant clinical improvement when combined with the potential prebiotic lactoferrin [85, 86]. Other recent studies have shown potentially beneficial effects of oral probiotics in plaque psoriasis [87, 88], adult AD [89], hand dermatitis [90], papulopustular rosacea, seborrhoeic dermatitis [86] and dandruff [91].

Although a number of studies have investigated the potential of oral probiotics, trials assessing the effect of topical probiotic and prebiotic interventions in skin disease are limited. Reduced AD disease severity in both adults and children treated with topical probiotics has been reported [92-94]. Further, interventional studies have observed reduced $S$. aureus colonization and one study even reported decreased $S$. aureus burden in patients after just a single application of a lotion containing antimicrobials isolated from human skin commensals [95]. In adult acne, a phase 2 clinical trial reported a significant reduction of acne severity and inflammatory lesions with the use of a topical probiotic spray, although this study seems yet to be published in full [96]. Individuals treated with a bacterial strain in an oil-inwater formulation displayed reductions in erythema and acne lesion size, along with reparation of the skin barrier [97]; another study reported improvement in skin health associated with a topical probiotic spray used in acne patients [98]. In addition, one RCT demonstrated that a single strain probiotic cream significantly reduced skin sensitivity and increased resistance to chemical and physical injury in females with reactive skin [99].

While some evidence exists to support the use of certain probiotic and/or prebiotic therapies in skin disease, the heterogeneity of outcomes between studies is a major limitation. This may be due to variations in treatment regimens, participant demographics, inclusion and exclusion criterion and the use of single versus multi-strain preparations, among others. Indeed, one study demonstrated the importance of strain choice on affecting the prevalence of AD [66]. Moreover, many studies are underpowered and limited by their small sample sizes and short follow-up. The transient nature of many skin diseases may necessitate longer studies to see true results. Only a few studies reported the ethnic background or skin type of participants, and even fewer commented on diet, even though host microbiome diversity associated with cultural and genetic factors is well appreciated [100]. Currently active and recruiting trials include investigations of oral probiotic supplementations in paediatric and adult AD, acne, as well as topical probiotics 


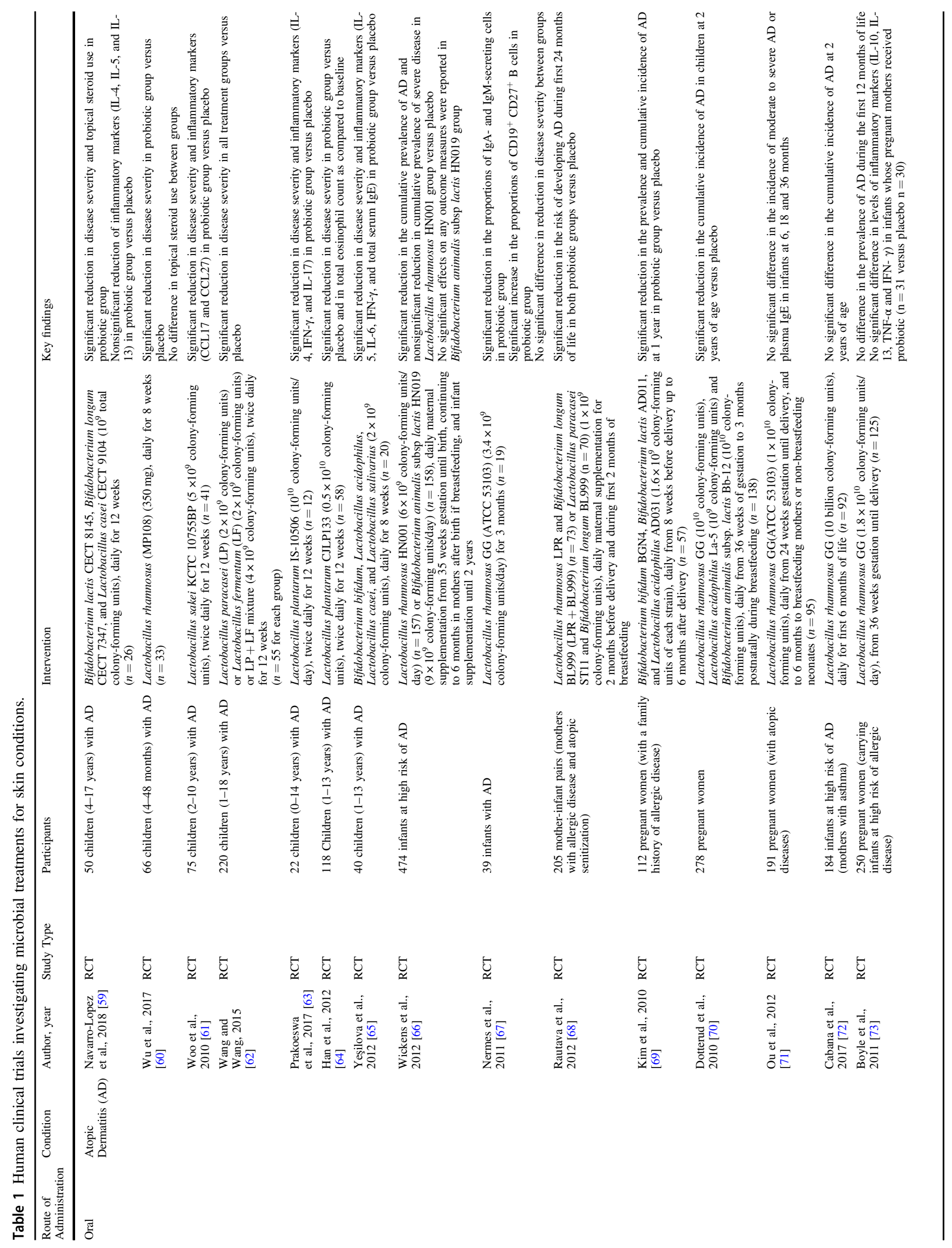




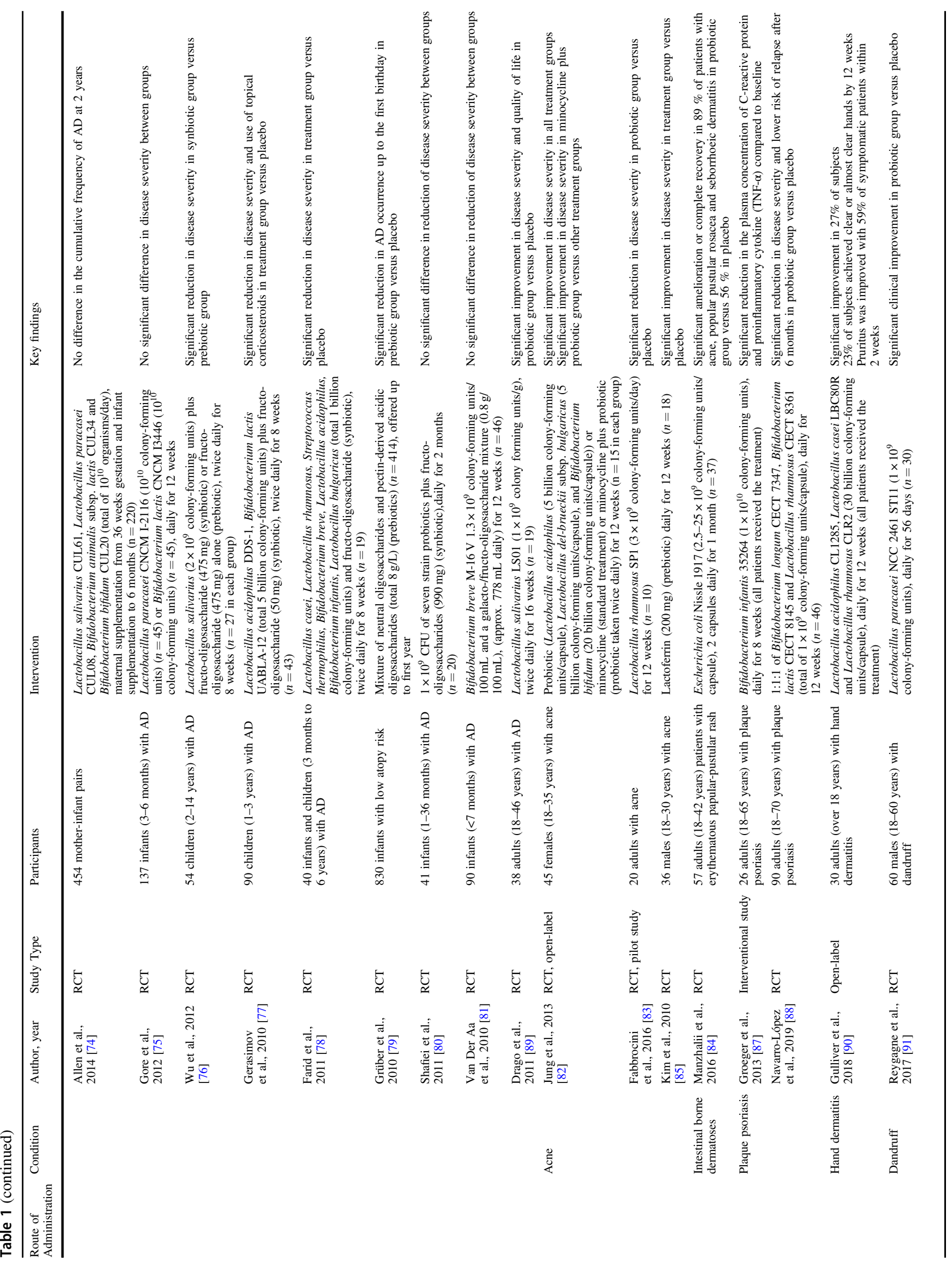




$$
\text { ind }
$$


in paediatric AD [101]. These studies and others will hopefully help to further clarify the usefulness of these interventions in skin disease, where microbial dysbiosis is a well-established pathogenic feature.

\section{Conclusions}

Significant advances have been made during recent years in describing the composition of the microbiome on the skin and the changes in bacterial communities that associate with, or sometimes precede, skin inflammatory disorders such as AD. However, substantial gaps in our knowledge on the microbiome still exist. In particular, the functional basis for microbe-host communication within the skin is still poorly described. In addition, novel probiotics and not just the traditional probiotic strains need to be clinically tested. Furthermore, microbial components or their metabolites should also be examined, in particular the application of these novel microbial drugs to the diseased site must be better explored.

Funding Open Access funding provided by the IReL Consortium.

\section{Compliance with ethical standards}

Conflict of interest The authors declare no competing interests.

Publisher's note Springer Nature remains neutral with regard to jurisdictional claims in published maps and institutional affiliations.

Open Access This article is licensed under a Creative Commons Attribution 4.0 International License, which permits use, sharing, adaptation, distribution and reproduction in any medium or format, as long as you give appropriate credit to the original author(s) and the source, provide a link to the Creative Commons license, and indicate if changes were made. The images or other third party material in this article are included in the article's Creative Commons license, unless indicated otherwise in a credit line to the material. If material is not included in the article's Creative Commons license and your intended use is not permitted by statutory regulation or exceeds the permitted use, you will need to obtain permission directly from the copyright holder. To view a copy of this license, visit http://creativecommons. org/licenses/by/4.0/.

\section{References}

1. Alkotob SS, Cannedy C, Harter K, Movassagh H, Paudel B, Prunicki $\mathrm{M}$, et al. Advances and novel developments in environmental influences on the development of atopic diseases. Allergy 2020;75:3077-86.

2. Kramer U, Sugiri D, Ranft U, Krutmann J, von Berg A, Berdel $\mathrm{D}$, et al. Eczema, respiratory allergies, and traffic-related air pollution in birth cohorts from small-town areas. J Dermatol Sci. 2009;56:99-105.

3. Lunjani N, Satitsuksanoa P, Lukasik Z, Sokolowska M, Eiwegger T, O'Mahony L. Recent developments and highlights in mechanisms of allergic diseases: microbiome. Allergy. 2018;73:2314-27.
4. Walter J, O'Mahony L. The importance of social networks-an ecological and evolutionary framework to explain the role of microbes in the aetiology of allergy and asthma. Allergy. 2019;74:2248-51.

5. Naik S, Bouladoux N, Linehan JL, Han SJ, Harrison OJ, Wilhelm C, et al. Commensal-dendritic-cell interaction specifies a unique protective skin immune signature. Nature. 2015; 520:104-8.

6. Lunjani N, Hlela C, O'Mahony L. Microbiome and skin biology. Curr Opin Allergy Clin Immunol. 2019;19:328-33.

7. Sabaté Brescó M, Harris LG, Thompson K, Stanic B, Morgenstern M, O'Mahony L, et al. Pathogenic mechanisms and host interactions in Staphylococcus epidermidis device-related infection. Front Microbiol. 2017;8:1401.

8. Belkaid Y, Segre JA. Dialogue between skin microbiota and immunity. Science. 2014;346:954-9.

9. Bosko CA. Skin barrier insights: from bricks and mortar to molecules and microbes. J Drugs Dermatol. 2019;18:63-67.

10. Grice EA, Segre JA. The skin microbiome. Nat Rev Microbiol. 2011;9:244-53.

11. Dréno B, Araviiskaia E, Berardesca E, Gontijo G, Sanchez Viera M, Xiang LF, et al. Microbiome in healthy skin, update for dermatologists. J Eur Acad Dermatol Venereol. 2016;30:2038-47.

12. Lange-Asschenfeldt B, Marenbach D, Lang C, Patzelt A, Ulrich M, Maltusch A, et al. Distribution of bacteria in the epidermal layers and hair follicles of the human skin. Ski Pharmacol Physiol. 2011;24:305-11.

13. Sanford JA, Gallo RL. Functions of the skin microbiota in health and disease. Semin Immunol. 2013;25:370-7.

14. Wang E, Qiang X, Li J, Zhu S, Wang P. The in vitro immunemodulating properties of a sweat gland-derived antimicrobial peptide dermcidin. Shock. 2016;45:28-32.

15. Byrd AL, Belkaid Y, Segre JA. The human skin microbiome. Nat Rev Microbiol. 2018;16:143-55.

16. Fleury OM, McAleer MA, Feuillie C, Formosa-Dague C, Sansevere E, Bennett DE, et al. Clumping factor B promotes adherence of Staphylococcus aureus to corneocytes in atopic dermatitis. Infect Immun. 2017;85:e00994-16.

17. Tordesillas L, Lozano-Ojalvo D, Dunkin D, Mondoulet L, Agudo J, Merad M, et al. PDL2(+) CD11b(+) dermal dendritic cells capture topical antigen through hair follicles to prime LAP (+) Tregs. Nat Commun. 2018;9:5238.

18. Scharschmidt TC, Vasquez KS, Pauli ML, Leitner EG, Chu K, Truong HA, et al. Commensal microbes and hair follicle morphogenesis coordinately drive treg migration into neonatal skin. Cell Host Microbe. 2017;21:467-77.

19. Lousada M, Lachnit T, Edelkamp J, Rouillé T, Ajdic D, Uchida $\mathrm{Y}$, et al. Exploring the human hair follicle microbiome. $\mathrm{Br} \mathrm{J}$ Dermatol. 2020. https://doi.org/10.1111/bjd.19461.

20. Nakatsuji T, Chiang HI, Jiang SB, Nagarajan H, Zengler K, Gallo RL. The microbiome extends to subepidermal compartments of normal skin. Nat Commun. 2013;4:1431.

21. Stubbendieck RM, May DS, Chevrette MG, Temkin MI, WendtPienkowski E, Cagnazzo J, et al. Competition among nasal bacteria suggests a role for siderophore-mediated interactions in shaping the human nasal microbiota. Appl Environ Microbiol. 2019;85:e02406-18.

22. Hardy BL, Dickey SW, Plaut RD, Riggins DP, Stibitz S, Otto M, et al. Corynebacterium pseudodiphtheriticum exploits Staphylococcus aureus virulence components in a novel polymicrobial defense strategy. mBio. 2019;10:e2491-18.

23. Stubbendieck RM, Vargas-Bautista C, Straight PD. Bacterial communities: interactions to scale. Front Microbiol. 2016; $7: 1234$.

24. Kwon S, Choi JY, Shin JW, Huh CH, Park KC, Du MH, et al. Changes in lesional and non-lesional skin microbiome during 
treatment of atopic dermatitis. Acta Derm Venereol. 2019; 99:284-90.

25. Kong HH, Oh J, Deming C, Conlan S, Grice EA, Beatson MA, et al. Temporal shifts in the skin microbiome associated with disease flares and treatment in children with atopic dermatitis. Genome Res. 2012;22:850-9.

26. Nakatsuji T, Chen TH, Narala S, Chun KA, Two AM, Yun T, et al. Antimicrobials from human skin commensal bacteria protect against Staphylococcus aureus and are deficient in atopic dermatitis. Sci Transl Med. 2017;9:eaah4680.

27. Iwase T, Uehara Y, Shinji H, Tajima A, Seo H, Takada K, et al. Staphylococcus epidermidis Esp inhibits Staphylococcus aureus biofilm formation and nasal colonization. Nature. 2010; 465:346-9.

28. Chen C, Krishnan V, Macon K, Manne K, Narayana SV, Schneewind O. Secreted proteases control autolysin-mediated biofilm growth of Staphylococcus aureus. J Biol Chem. 2013; 288:29440-52.

29. Zipperer A, Konnerth MC, Laux C, Berscheid A, Janek D, Weidenmaier $\mathrm{C}$, et al. Human commensals producing a novel antibiotic impair pathogen colonization. Nature. 2016;535:511-6.

30. Francuzik W, Franke K, Schumann RR, Heine G, Worm M. Propionibacterium acnes Abundance correlates inversely with Staphylococcus aureus: data from atopic dermatitis skin microbiome. Acta Derm Venereol. 2018;98:490-5.

31. Paharik AE, Parlet CP, Chung N, Todd DA, Rodriguez EI, Van Dyke MJ, et al. Coagulase-negative Staphylococcal strain prevents Staphylococcus aureus colonization and skin infection by blocking quorum sensing. Cell Host Microbe. 2017;22:746-56.

32. Peng P, Baldry M, Gless BH, Bojer MS, Espinosa-Gongora C, Baig SJ, et al. Effect of co-inhabiting coagulase negative Staphylococci on $S$. aureus agr quorum sensing, host factor binding, and biofilm formation. Front Microbiol. 2019;10:2212.

33. Glatthardt T, Campos JCM, Chamon RC, de Sá Coimbra TF, Rocha GA, de Melo MAF, et al. Small molecules produced by commensal Staphylococcus epidermidis disrupt formation of biofilms by Staphylococcus aureus. Appl Environ Microbiol. 2020;86:e02539-19.

34. Baldry M, Nakamura Y, Nakagawa S, Frees D, Matsue H, Nunez $\mathrm{G}$, et al. Application of an agr-specific antivirulence compound as therapy for Staphylococcus aureus-induced inflammatory skin disease. J Infect Dis. 2018;218:1009-13.

35. Cau L, Williams MR, Butcher AM, Nakatsuji T, Kavanaugh JS, Cheng JY, et al. Staphylococcus epidermidis protease EcpA can be a deleterious component of the skin microbiome in atopic dermatitis. J Allergy Clin Immunol. 2021;147:955-66.

36. O'Neill AM, Nakatsuji T, Hayachi A, Williams MR, Mills RH, Gonzalez DJ, et al. Identification of a human skin commensal bacterium that selectively kills Cutibacterium acnes. J Investig Dermatol 2020;140:1619-28.

37. Huang YJ, Marsland BJ, Bunyavanich S, O’Mahony L, Leung DY, Muraro A, et al. The microbiome in allergic disease: current understanding and future opportunities. J Allergy Clin Immunol. 2017;139:1099-110.

38. Casterline B, Paller A. Early development of the skin microbiome: therapeutic opportunities. Pediatr Res. 2020; https://doi. org/10.1038/s41390-020-01146-2.

39. Santoro A, Zhao J, Wu L, Carru C, Biagi E, Franceschi C. Microbiomes other than the gut: inflammaging and age-related diseases. Semin Immunopathol. 2020;42:589-605.

40. Bjerre RD, Bandier J, Skov L, Engstrand L, Johansen JD. The role of the skin microbiome in atopic dermatitis: a systematic review. Br J Dermatol. 2017;177:1272-8.

41. Scharschmidt TC, Vasquez KS, Pauli ML, Leitner EG, Chu K, Truong HA, et al. Commensal microbes and hair follicle morphogenesis coordinately drive treg migration into neonatal skin. Cell Host Microbe. 2017;21:467-77.

42. Grönroos M, Parajuli A, Laitinen O, Roslund M, Vari H, Hyöty $\mathrm{H}$, et al. Short-term direct contact with soil and plant materials leads to an immediate increase in diversity of skin microbiota. MicrobiologyOpen 2019;8:e0645.

43. Roslund M, Puhakka R, Grönroos M, Nurminen N, Oikarinen S, Gazali A, et al. Biodiversity intervention enhances immune regulation and health-associated commensal microbiota among daycare children. Sci Adv. 2020;6:eaba2578.

44. Sun L, Liu W, Zhang LJ. The role of toll-like receptors in skin host defense, psoriasis, and atopic dermatitis. J Immunol Res. 2019:1824624. https://doi.org/10.1155/2019/1824624.

45. Borriello F, Zanoni I, Granucci F. Cellular and molecular mechanisms of antifungal innate immunity at epithelial barriers: the role of C-type lectin receptors. Eur J Immunol. 2020;50:317-25.

46. Leśniak W, Graczyk-Jarzynka A. The S100 proteins in epidermis: topology and function. Biochim Biophys Acta. 2015;1850:2563-72.

47. Geoghegan JA, Irvine AD, Foster TJ. Staphylococcus aureus and atopic dermatitis: a complex and evolving relationship. Trends Microbiol. 2018;26:484-97.

48. Heath W, Carbone F. The skin-resident and migratory immune system in steady state and memory: innate lymphocytes, dendritic cells and T cells. Nat Immunol. 2013;14:978-85.

49. Hussain M, Borcard L, Walsh KP, Rodriguez MP, Mueller C, Kim BS, et al. Basophil-derived IL-4 promotes epicutaneous antigen sensitization concomitant with the development of food allergy. J Allergy Clin Immunol. 2018;141:223-34.

50. Eiwegger T, Hung L, San Diego KE, O'Mahony L, Upton J. Recent developments and highlights in food allergy. Allergy 2019;74:2355-67.

51. Ottman N, Barrientos-Somarribas M, Fyhrquist N, Alexander H, Wisgrill L, Olah $\mathrm{P}$, et al. Microbial and transcriptional differences elucidate atopic dermatitis heterogeneity across skin sites. Allergy. 2020. https://doi.org/10.1111/all.14606.

52. Yanez D, Lacher R, Vidyarthi A, Colegio O. The role of macrophages in skin homeostasis. Pflügers Arch. 2017;469:455-63.

53. Wang Z, Mascarenhas N, Eckmann L, Miyamoto Y, Sun X, Kawakami T, et al. Skin microbiome promotes mast cell maturation by triggering stem cell factor production in keratinocytes. J Allergy Clin Immunol. 2017;139:1205-16.

54. Igawa S, Di Nardo A. Skin microbiome and mast cells. Transl Res. 2017;184:68-76.

55. Legoux F, Salou M, Lantz O. MAIT cell development and functions: the microbial connection. Immunity. 2020;53:710-23.

56. Lin Q, Kuypers M, Philpott DJ, Mallevaey T. The dialogue between unconventional $\mathrm{T}$ cells and the microbiota. Mucosal Immunol. 2020;13:867-76.

57. Nel I, Bertrand L, Toubal A, Lehuen A. MAIT cells, guardians of skin and mucosa? Mucosal Immunol. 2021:1-12. https://doi. org/10.1038/s41385-021-00391-w.

58. Legoux F, Salou M, Lantz O. MAIT cell development and functions: the microbial connection. Immunity. 2020;53:710-23.

59. Navarro-Lopez V, Ramirez-Bosca A, Ramon-Vidal D, RuzafaCostas B, Genoves-Martinez S, Chenoll-Cuadros E, et al. Effect of oral administration of a mixture of probiotic strains on SCORAD index and use of topical steroids in young patients with moderate atopic dermatitis a randomized clinical trial. JAMA Dermatol. 2018;154:37-43.

60. Wu YJ, Wu WF, Hung CW, Ku MS, Liao PF, Sun HL, et al. Evaluation of efficacy and safety of Lactobacillus rhamnosus in children aged 4-48 months with atopic dermatitis: an 8-week, double-blind, randomized, placebo-controlled study. J Microbiol Immunol Infect. 2017;50:684-92. 
61. Il WooS, Kim JY, Lee YJ, Kim NS, Hahn YS. Effect of Lactobacillus sakei supplementation in children with atopic eczemadermatitis syndrome. Ann Allergy, Asthma Immunol. 2010; 104:343-8.

62. Wang IJ, Wang JY. Children with atopic dermatitis show clinical improvement after Lactobacillus exposure. Clin Exp Allergy. 2015;45:779-87.

63. Prakoeswa CRS, Herwanto N, Prameswari R, Astari L, Sawitri S, Hidayati AN, et al. Lactobacillus plantarum IS-10506 supplementation reduced SCORAD in children with atopic dermatitis. Benef Microbes. 2017;8:833-40.

64. Han Y, Kim B, Ban J, Lee J, Kim BJ, Choi BS, et al. A randomized trial of Lactobacillus plantarum CJLP133 for the treatment of atopic dermatitis. Pediatr Allergy Immunol. 2012;23:667-73.

65. Yeşilova Y, Çalka Ö, Akdeniz N, Berktaş M. Effect of probiotics on the treatment of children with atopic dermatitis. Ann Dermatol. 2012;24:189-93.

66. Wickens K, Black P, Stanley TV, Mitchell E, Barthow C, Fitzharris $\mathrm{P}$, et al. A protective effect of Lactobacillus rhamnosus HN001 against eczema in the first 2 years of life persists to age 4 years. Clin Exp Allergy. 2012;42:1071-9.

67. Nermes M, Kantele JM, Atosuo TJ, Salminen S, Isolauri E. Interaction of orally administered Lactobacillus rhamnosus GG with skin and gut microbiota and humoral immunity in infants with atopic dermatitis. Clin Exp Allergy. 2011;41:370-7.

68. Rautava S, Kainonen E, Salminen S, Isolauri E. Maternal probiotic supplementation during pregnancy and breast-feeding reduces the risk of eczema in the infant. J Allergy Clin Immunol. 2012;30:1355-60.

69. Kim JY, Kwon JH, Ahn SH, Lee S IL, Han YS, Choi YO, et al. Effect of probiotic mix (Bifidobacterium bifidum, Bifidobacterium lactis, Lactobacillus acidophilus) in the primary prevention of eczema: a double-blind, randomized, placebo-controlled trial. Pediatr Allergy Immunol. 2010;21:386-93.

70. Dotterud CK, Storrø O, Johnsen R, Øien T. Probiotics in pregnant women to prevent allergic disease: a randomized, doubleblind trial. Br J Dermatol. 2010;163:616-23.

71. Ou CY, Kuo HC, Wang L, Hsu TY, Chuang H, Liu CA, et al. Prenatal and postnatal probiotics reduces maternal but not childhood allergic diseases: a randomized, double-blind, placebo-controlled trial. Clin Exp Allergy. 2012;42:1386-96.

72. Cabana MD, McKean M, Caughey AB, Fong L, Lynch S, Wong A, et al. Early probiotic supplementation for eczema and asthma prevention: a randomized controlled trial. Pediatrics. 2017;140: e20163000.

73. Boyle RJ, Ismail IH, Kivivuori S, Licciardi PV, Robins-Browne $\mathrm{RM}, \mathrm{Mah} \mathrm{LJ}$, et al. Lactobacillus GG treatment during pregnancy for the prevention of eczema: a randomized controlled trial. Allergy. 2011;66:509-16.

74. Allen SJ, Jordan S, Storey M, Thornton CA, Gravenor MB, Garaiova I, et al. Probiotics in the prevention of eczema: a randomised controlled trial. Arch Dis Child. 2014;99:1014-9.

75. Gore C, Custovic A, Tannock GW, Munro K, Kerry G, Johnson $\mathrm{K}$, et al. Treatment and secondary prevention effects of the probiotics Lactobacillus paracasei or Bifidobacterium lactis on early infant eczema: randomized controlled trial with follow-up until age 3 years. Clin Exp Allergy. 2012;42:112-22.

76. Wu KG, Li TH, Peng HJ. Lactobacillus salivarius plus fructooligosaccharide is superior to fructo-oligosaccharide alone for treating children with moderate to severe atopic dermatitis: a double-blind, randomized, clinical trial of efficacy and safety. $\mathrm{Br}$ J Dermatol. 2012;166:129-36.

77. Gerasimov SV, Vasjuta VV, Myhovych OO, Bondarchuk LI. Probiotic supplement reduces atopic dermatitis in preschool children. Am J Clin Dermatol. 2010;11:351-61.
78. Farid R, Ahanchian H, Jabbari F, Moghiman T. Effect of a new synbiotic mixture on atopic dermatitis in children: a randomizedcontrolled trial. Iran J Pediatr. 2011;21:225-30.

79. Grüber C, Van Stuijvenberg M, Mosca F, Moro G, Chirico G, Braegger CP, et al. Reduced occurrence of early atopic dermatitis because of immunoactive prebiotics among low-atopy-risk infants. J Allergy Clin Immunol. 2010;126:791-7.

80. Shaflei A, Moin M, Pourpak Z, Gharagozlou M, Aghamohamadi A, Sajedi V, et al. Synbiotics could not reduce the Scoring of Childhood Atopic Dermatitis (SCORAD): a randomized double blind placebo-controlled trial. Iran J Allergy, Asthma Immunol. 2011;10:21-28.

81. Van Der Aa LB, Heymans HS, Van Aalderen WM, Sillevis Smitt JH, Knol J, Ben Amor K, et al. Effect of a new synbiotic mixture on atopic dermatitis in infants: a randomized-controlled trial. Clin Exp Allergy. 2010;40:795-804.

82. Jung GW, Tse JE, Guiha I, Rao J. Prospective, randomized, open-label trial comparing the safety, efficacy, and tolerability of an acne treatment regimen with and without a probiotic supplement and minocycline in subjects with mild to moderate acne. J Cutan Med Surg. 2013;17:114-22.

83. Fabbrocini G, Bertona M, Picazo, Pareja-Galeano H, Monfrecola G, Emanuele E. Supplementation with Lactobacillus rhamnosus SP1 normalises skin expression of genes implicated in insulin signalling and improves adult acne. Benef Microbes. 2016;7:625-30.

84. Manzhalii E, Hornuss D, Stremmel W. Intestinal-borne dermatoses significantly improved by oral application of Escherichia coli Nissle 1917. World J Gastroenterol. 2016;22:5415-21.

85. Kim J, Ko Y, Park YK, Kim NI, Ha WK, Cho Y. Dietary effect of lactoferrin-enriched fermented milk on skin surface lipid and clinical improvement of acne vulgaris. Nutrition. 2010;26:902-9.

86. Chen PW, Liu ZS, Kuo TC, Hsieh MC, Li ZW. Prebiotic effects of bovine lactoferrin on specific probiotic bacteria. BioMetals. 2017;30:237-48.

87. Groeger D, O'Mahony L, Murphy EF, Bourke JF, Dinan TG, Kiely $\mathrm{B}$, et al. Bifidobacterium infantis 35624 modulates host inflammatory processes beyond the gut. Gut Microbes. 2013;4:325-39.

88. Navarro-López V, Martínez-Andrés A, Ramírez-Boscà A, Ruzafa-Costas B, Núñez-Delegido E, Carrión-Gutiérrez MA, et al. Efficacy and safety of oral administration of a mixture of probiotic strains in patients with psoriasis: a randomized controlled clinical trial. Acta Derm Venereol. 2019;99:1078-84.

89. Drago L, Iemoli E, Rodighiero V, Nicola L, De Vecchi E, Piconi S. Effects of Lactobacillus salivarius LS01 (DSM 22775) treatment on adult atopic dermatitis: a randomized placebo-controlled study. Int J Immunopathol Pharmacol. 2011;24:1037-48.

90. Gulliver WP, Hutton AS, Ship N. Investigating the therapeutic potential of a probiotic in a clinical population with chronic hand dermatitis. Clin Cosmet Investig Dermatol. 2018;11:265-71.

91. Reygagne P, Bastien P, Couavoux MP, Philippe D, Renouf M, Castiel-Higounenc I, et al. The positive benefit of Lactobacillus paracasei NCC2461 ST11 in healthy volunteers with moderate to severe dandruff. Benef Microbes. 2017;8:671-80.

92. Blanchet-Réthoré $\mathrm{S}$, Bourdès $\mathrm{V}$, Mercenier $\mathrm{A}$, Haddar $\mathrm{CH}$, Verhoeven PO, Andres P. Effect of a lotion containing the heattreated probiotic strain Lactobacillus johnsonii NCC 533 on Staphylococcus aureus colonization in atopic dermatitis. Clin Cosmet Investig Dermatol. 2017;10:249-57.

93. Myles IA, Earland NJ, Anderson ED, Moore IN, Kieh MD, Williams KW, et al. First-in-human topical microbiome transplantation with Roseomonas mucosa for atopic dermatitis. JCI insight. 2018;3:e120608.

94. Butler É, Lundqvist C, Axelsson J. Lactobacillus reuteri DSM 17938 as a novel topical cosmetic ingredient: a proof of concept clinical study in adults with atopic dermatitis. Microorganisms. 2020;8:1026. 
95. Nakatsuji T, Chen TH, Narala S, Chun KA, Two AM, Yun T, et al. Antimicrobials from human skin commensal bacteria protect against Staphylococcus aureus and are deficient in atopic dermatitis. Sci Transl Med. 2017;9:eaah4680.

96. Craft N. Clinical trial in subjects with mild to moderate acne vulgaris. ClinicalTrials.gov Identifier: NCT02832063; 2017.

97. Muizzuddin N, Maher W, Sullivan M, Schnittger S, Mammone T. Physiological effect of a probiotic on skin. J Cosmet Sci. 2012;63:385-95.

98. Bateni E, Tester R, Al-Ghazzewi F, Bateni S, Alvani K, Piggott J. The use of Konjac Glucomannan Hydrolysates (GMH) to improve the health of the skin and reduce acne vulgaris. Am J Dermatol Venereol. 2013;2:10-14.

99. Guéniche A, Bastien P, Ovigne JM, Kermici M, Courchay G, Chevalier V, et al. Bifidobacterium longum lysate, a new ingredient for reactive skin. Exp Dermatol. 2010;19:e1-8.

100. Chen YE, Tsao H. The skin microbiome: current perspectives and future challenges. J Am Acad Dermatol. 2013;69:143-55.

101. Clinicaltrials.gov. Probiotics I Skin Disease I Recruiting, Active, not recruiting. https://clinicaltrials.gov/ct2/results?term= probiotic $\&$ cond $=$ Skin + Diseases $\&$ recrs $=\mathrm{a} \&$ recrs $=\mathrm{f} \&$ recrs $=\mathrm{d} \& \mathrm{a}$ ge $\_\mathrm{v}=\&$ gndr $=\&$ type $=\& \mathrm{rslt}=\&$ Search $=$ Apply. 